\title{
Effect of Initial Stress on the Propagation Characteristics of Waves in Fiber-Reinforced Transversely Isotropic Thermoelastic Material under an Inviscid Liquid Layer
}

\author{
Rajneesh Kumar, ${ }^{1}$ Sanjeev Ahuja, ${ }^{2}$ and S. K. Garg ${ }^{3}$ \\ ${ }^{1}$ Department of Mathematics, Kurukshetra University, Kurukshetra, Haryana 136119, India \\ ${ }^{2}$ University Institute of Engg. \& Tech., Kurukshetra University, Kurukshetra, Haryana 136119, India \\ ${ }^{3}$ Department of Mathematics, Deen Bandhu Chotu Ram Uni. of Sc. \& Tech., Sonipat, Haryana 131027, India \\ Correspondence should be addressed to Sanjeev Ahuja; sanjeev_ahuja81@hotmail.com
}

Received 24 May 2014; Accepted 17 July 2014; Published 26 August 2014

Academic Editor: Felix Sharipov

Copyright (C) 2014 Rajneesh Kumar et al. This is an open access article distributed under the Creative Commons Attribution License, which permits unrestricted use, distribution, and reproduction in any medium, provided the original work is properly cited.

\begin{abstract}
The present investigation deals with the propagation of waves in fiber-reinforced transversely isotropic thermoelastic solid half space with initial stresses under a layer of inviscid liquid. The secular equation for surface equation in compact form is derived after developing the mathematical model. The phase velocity and attenuation coefficients of plane waves are studied numerically for a particular model. Effects of initial stress and thickness of the layer on the phase velocity, attenuation coefficient, and specific loss of energy are predicted graphically in the certain model. A particular case of Rayleigh wave has been discussed and the dispersion curves of the phase velocity and attenuation coefficients have also been presented graphically. Some other particular cases are also deduced from the present investigation.
\end{abstract}

\section{Introduction}

Fiber-reinforced materials are widely used in engineering structures due to their superiority over the structural materials in applications requiring high strength and stiffness in lightweight components. Consequently, characterization of their mechanical behavior is of particular importance for structural design using these materials. Fibers are assumed an inherent material property, rather than some form of inclusion in models as Spencer [1]. In the case of an elastic solid reinforced by a series of parallel fibers it is usual to assume transverse isotropy.

The idea of continuous self-reinforcement at every point of an elastic solid was introduced by Belfield et al. [2]. The characteristic property of reinforced concrete member is that its components, namely, concrete and steel, act together as a single anisotropic unit as main long as they remain in the elastic condition; that is, the two components are bound together so that there can be no relative displacement between them. The dynamical interaction between the thermal and mechanical fields in solids has great practical applications in modern aeronautics, astronautics, nuclear reactors, and high energy particle accelerators.

The analysis of stress and deformation of fiber-reinforced composite materials has been an important subject of solid mechanics for last three decades. Pipkin [3] did pioneer works on the subject. Sengupta and Nath [4] discussed the problem of surface waves in fiber-reinforced anisotropic elastic media.

Lord and Shulman [5] introduced a theory of generalized thermoelasticity with one relaxation time for an isotropic body. The theory was extended for anisotropic body by Dhaliwal and Sherief [6]. In this theory, a modified law of heat conduction including both the heat flux and its time derivatives replaces the conventional Fourier's Law. The heat equation associated with this theory is hyperbolic and hence eliminates the paradox of infinite speeds of propagation inherent in both coupled and uncoupled theories of thermoelasticity. Erdem [7] derived heat conduction equation for a composite rigid material containing an arbitrary distribution of fibers. Recently, Kumar and Gupta [8] discussed the Wave motion in an anisotropic fiber-reinforced thermoelastic solid. 
Surface waves carry a lot of information about the Earth's crust and dispersion analysis of surface waves is concerned with the phase velocity and wave number. Further the velocity of seismic waves depends upon the elastic parameters of the medium through which they travel. Therefore, many investigators have studied the surface wave propagation by considering different kinds of models. Chadwick and Seet [9] and, Singh and Sharma [10] have discussed the propagation of plane harmonic waves in transversely isotropic thermoelastic materials. Singh [11] studied a problem on wave propagation in an anisotropic generalized thermoelastic solid and obtained a cubic equation, which gives the dimensional velocities of various plane waves.

Chadwick [12] has discussed the propagation of surface waves in homogeneous thermoelastic media. Chadwick and Windle [13] studied the effect of heat conduction on the propagation of Rayleigh waves in the semi-infinite media (i) when the surface is maintained at constant temperature and (ii) when the surface is thermally insulated. Sharma and Singh [14] have studied thermoelastic surface waves in a transversely isotropic half-space.

The study of wave propagation in a generalized thermoelastic media with additional parameters like prestress, porosity, viscosity, microstructure, temperature, and other parameters provide vital information about existence of new or modified waves. Such information may be useful for experimental seismologists in correcting earthquake estimation.

Prestressed materials have various applications, for example, in oil and geophysical industry, NDT in prestressed materials, use of rubber composites in automotive, and aerospace and defense industries (often in prestressed states) and in the study of biological tissues (lung, tendon, etc.) which are all nonlinear prestressed viscoelastic composites.

Montanaro [15] investigated the isotropic linear thermoelasticity with hydrostatic initial stress. Wang and Slattery [16] formulated the thermoelastic equations without energy dissipation for a body which has previously received a large deformation and is at nonuniform temperature. Ieşan [17] presented a theory of Cosserat thermoelastic solids with initial stresses. Ames and Straughan [18] derived the continuous dependence results for initially prestressed thermoelastic bodies.

Some theorems in the generalized theory of thermoelasticity for prestressed bodies were studied by Wang et al. [19]. Marin and Marinescu [20] studied the asymptotic partition of total energy for the solutions of the mixed initial boundary value problem within the context of the thermoelasticity of initially stressed bodies. Kalinchuk et al. [21] studied the problem of steady-state harmonic oscillations for a nonhomogeneous thermoelastic prestressed medium.

Othman and Song [22] discussed the reflection of plane waves from a thermoelastic elastic solid half-space under hydrostatic initial stress without energy dissipation. Singh [23] studied wave propagation in an initially stressed transversely isotropic thermoelastic half-space. However, no attempt has been made to discuss the effect of hydrostatic initial stress on propagation of waves in the layer of fiberreinforced transversely isotropic thermoelastic material. Othman et al. [24] investigated the influences of fractional order, hydrostatic initial stress, and gravity field on the plane waves in a fiber-reinforced isotropic thermoelastic medium using normal mode analysis. Abd-Alla et al. [25] studied the effects of rotation and gravity field on surface waves in fiber-reinforced thermoelastic media under four theories. Othman and Atwa [26] studied the effect of rotation, gravity, and reinforcement on the total deformation and mutual interaction of the body in a fiber-reinforced thermoelastic solid using normal mode analysis in the context of GreenNaghdi theory of thermoelasitcity. Abbas [27] studied the transient phenomena in the magnetothermoelastic model in the context of the Lord and Shulman theory in a perfectly conducting medium. Abbas [28] constructed the equations for generalized thermoelasticity of an unbounded fiberreinforced anisotropic medium with a circular hole in the context of Green and Naghdi (GN) theory. Recently, Abbas and Zenkour [29] investigated two-temperature generalized thermoelasticity with two relaxation times for an infinite fiber-reinforced anisotropic plate containing a circular cavity using a finite element method.

In the present paper, the governing equations of thermoelastcity of fiber-reinforced transversely isotropic solid half space with initial stresses under a layer of inviscid liquid are formulated and solved analytically in two dimensions at uniform temperature. The phase velocity and attenuation coefficients of plane waves are studied numerically for a particular model. Specific loss of energy is obtained and discussed numerically for a particular model to study the effects of initial stress. The study of present investigation has applications in civil engineering and geophysics.

\section{Basic Equations}

The basic equations in the dynamic theory of the homogeneous thermally conducting fiber-reinforced medium with an initial hydrostatic stress without body forces and heat sources are given by Lord and Shulman [5] and Abbas and Othman [30] as

$$
\sigma_{i j, j}-P \omega_{i j, j}=\rho \ddot{u}_{i}, \quad i, j=1,2,3
$$

and heat conduction equation is given by

$$
K_{i j} T_{, i j}=\left(\frac{\partial}{\partial t}+\tau_{0} \frac{\partial^{2}}{\partial t^{2}}\right)\left(T_{o} \beta_{i j} \dot{u}_{i, j}+\rho C_{e} \dot{T}\right), \quad i, j=1,2,3 .
$$

The constitutive relations for thermally conducting transversely isotropic, fiber-reinforced linearly elastic media are

$$
\begin{gathered}
\sigma_{i j}=\lambda e_{k k} \delta_{i j}+2 \mu_{T} e_{i j}+\alpha\left(a_{k} a_{m} e_{k m} \delta_{i j}+a_{i} a_{j} e_{k k}\right) \\
+2\left(\mu_{L}-\mu_{T}\right)\left(a_{i} a_{k} e_{k j}+a_{j} a_{k} e_{k i}\right) \\
+\beta\left(a_{k} a_{m} e_{k m} a_{i} a_{j}\right)-\beta_{i j} T \delta_{i j}, \\
i, j, k, m=1,2,3,
\end{gathered}
$$


where

$$
\begin{array}{r}
e_{i j}=\frac{1}{2}\left(u_{i, j}+u_{j, i}\right), \quad \omega_{i j}=\frac{1}{2}\left(u_{j, i}-u_{i, j}\right), \\
i, j=1,2,3,
\end{array}
$$

and $\lambda, \mu_{T}$ are elastic parameters, $\alpha, \beta,\left(\mu_{L}-\mu_{T}\right)$ are reinforced anisotropic elastic parameters, $\rho$ is the mass density, $\sigma_{i j}$ are components of stress tensor, $u_{i}$ are the displacement components, $e_{i j}$ are components of infinitesimal strain, $T$ is the temperature change of a material particle, $T_{o}$ is the reference uniform temperature of the body, $k_{i j}$ are coefficients of thermal conductivity, $\beta_{i j}$ are thermal elastic coupling tensor, $C_{e}$ is the specific heat at constant strain, $\delta_{i j}$ is the Kronecker delta, and $P$ is the initial Pressure. The comma notation is used for spatial derivatives and superimposed dot represents time differentiation. $a_{j}$ are components of $\mathbf{a}$, all referred to Cartesian coordinate. The vector a may be a function of position. We choose a so that its components are $(1,0,0)$.

The equation which governs the motion in terms of velocity potential $\psi$ of the homogeneous inviscid liquid is given by Ewing et al. [31]:

$$
\frac{\partial^{2} \psi}{\partial x_{1}^{2}}+\frac{\partial^{2} \psi}{\partial x_{2}^{2}}=\frac{1}{\left(\alpha^{\ell}\right)^{2}} \frac{\partial^{2} \psi}{\partial t^{2}},
$$

where $\alpha^{\ell}=\sqrt{\lambda^{\ell} / \rho^{\ell}}$ is the velocity of sound liquid.

The displacement components $u_{1}^{\ell}, u_{2}^{\ell}$ and pressure $p^{\ell}$ in the medium $M_{2}$ are given by

$$
u_{1}^{\ell}=\frac{\partial \psi}{\partial x_{1}}, \quad u_{2}^{\ell}=\frac{\partial \psi}{\partial x_{2}}, \quad p^{\ell}=-\rho^{\ell} \frac{\partial \psi}{\partial t} .
$$

\section{Formulation of the Problem}

We consider a homogeneous, thermally conducting transversely isotropic fiber-reinforced medium $\left(M_{1}\right)$ with an initial hydrostatic stress lying under a uniform homogeneous inviscid liquid layer medium $\left(M_{2}\right)$ of thickness $H$. We take the rectangular Cartesian coordinate system $\mathrm{Ox}_{1} x_{2} x_{3}$ at any point on the plane horizontal surface and $x_{1}$-axis in the direction of wave propagation and $x_{2}$-axis pointing vertically downward into the half-space so that all particles on a line parallel to $x_{3}$-axis are equally displaced; therefore, all the field quantities will be independent of $x_{3}$-coordinates. Hence the liquid layer medium $M_{2}$ occupies the region $-H<x_{2}<0$ and $x_{2}>0$ is occupied by the half-space medium $M_{1}$. The plane $x_{2}=-H$ represents the free surface of the liquid and $x_{2}=0$ is taken as interface between $M_{1}$ and $M_{2}$ medium (Figure 1).

The displacement components for medium $M_{1}$ are taken as

$$
\vec{u}=\left(u_{1}, u_{2}, 0\right)
$$

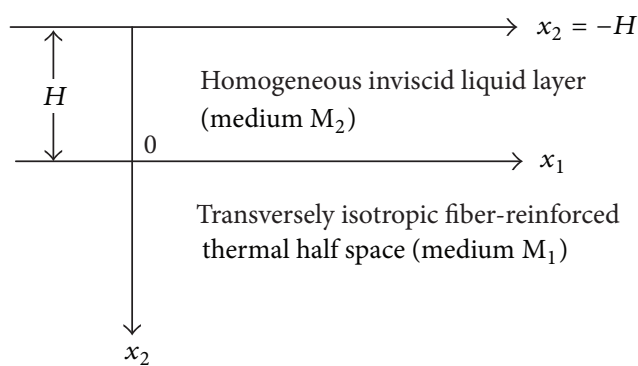

Figure 1: Geometry of the problem.

With the help of (3), (4), and (7) and (1)-(2), take the form

$$
\begin{aligned}
& C_{11} \frac{\partial^{2} u_{1}}{\partial x_{1}^{2}}+\left(C_{12}+C_{0}-\frac{P}{2}\right) \frac{\partial^{2} u_{2}}{\partial x_{1} \partial x_{2}} \\
& +\left(C_{0}+\frac{P}{2}\right) \frac{\partial^{2} u_{1}}{\partial x_{2}^{2}}-\beta_{11} \frac{\partial T}{\partial x_{1}}=\rho \frac{\partial^{2} u_{1}}{\partial t^{2}},
\end{aligned}
$$

$$
+\left(C_{0}+\frac{P}{2}\right) \frac{\partial^{2} u_{2}}{\partial x_{1}^{2}}-\beta_{22} \frac{\partial T}{\partial x_{2}}=\rho \frac{\partial^{2} u_{2}}{\partial t^{2}},
$$

$$
\begin{aligned}
& \frac{\partial^{2} T}{\partial x_{1}^{2}}+\bar{k} \frac{\partial^{2} T}{\partial x_{2}^{2}}-\frac{\rho C_{e}}{k_{11}}\left(\frac{\partial T}{\partial t}+\tau_{o} \frac{\partial^{2} T}{\partial t^{2}}\right) \\
& \quad=\varepsilon\left[\left(\frac{\partial^{2} u_{1}}{\partial x_{1} \partial t}+\tau_{o} \frac{\partial^{3} u_{1}}{\partial x_{1} \partial t^{2}}\right)+\bar{\beta}\left(\frac{\partial^{2} u_{2}}{\partial x_{2} \partial t}+\tau_{o} \frac{\partial^{3} u_{2}}{\partial x_{2} \partial t^{2}}\right)\right],
\end{aligned}
$$

where

$$
\begin{gathered}
\beta_{11}=\left(C_{11}+C_{12}\right) \alpha_{11}+C_{12} \alpha_{22}, \\
\beta_{22}=\left(C_{12}+C_{23}-C_{55}\right) \alpha_{11}+C_{22} \alpha_{22}, \\
C_{12}=\lambda+\alpha, \quad C_{11}=\lambda+2 \alpha+4 \mu_{L}-2 \mu_{T}+\beta, \\
C_{22}=C_{33}=\lambda+2 \mu_{T}, \\
C_{44}=C_{66}=2 \mu_{L}, \quad C_{55}=2 \mu_{T}, \\
C_{23}=C_{33}-C_{55}, \quad C_{o}=\frac{C_{44}}{2}, \\
\varepsilon=\frac{T_{o} \beta_{11}}{k_{11}}, \quad \bar{k}=\frac{k_{22}}{k_{11}}, \quad \bar{\beta}=\frac{\beta_{22}}{\beta_{11}},
\end{gathered}
$$

and $\lambda, \alpha, \beta, \mu_{L}, \mu_{T}$ are material constants, $\alpha_{11}, \alpha_{22}$ are components of linear thermal expansion, and $\tau_{o}$ is thermal relaxation time. 
To facilitate the solution, the following dimensionless quantities are introduced:

$$
\begin{gathered}
\left(x_{1}^{\prime}, x_{2}^{\prime}\right)=\frac{w^{*}}{v_{1}}\left(x_{1}, x_{2}\right), \quad\left(u_{1}^{\prime}, u_{2}^{\prime}\right)=\frac{\rho v_{1} w^{*}}{\beta_{11} T_{o}}\left(u_{1}, u_{2}\right), \\
t_{i j}^{\prime}=\frac{t_{i j}}{\beta_{11} T_{o}}, \quad T^{\prime}=\frac{T}{T_{o}}, \quad t^{\prime}=w^{*} t, \\
h^{\prime}=\frac{v_{1}}{w^{*}} h, \quad \tau_{o}^{\prime}=w^{*} \tau_{o}, \quad \psi^{\prime}=\frac{w^{*} \psi}{v_{1}^{2}}, \\
\left(u_{1}^{\ell^{\prime}}, u_{2}^{\ell^{\prime}}\right)=\frac{w^{*}}{v_{1}}\left(u_{1}^{\ell}, u_{2}^{\ell}\right), \quad p^{\ell^{\prime}}=\frac{p^{\ell}}{\lambda^{\ell}}, \quad t_{22}^{\ell^{\prime}}=\frac{t_{22}^{\ell}}{\lambda^{\ell}},
\end{gathered}
$$

where $w^{*}=C_{e} C_{11} / k_{11}, v_{1}^{2}=C_{11} / \rho$ and $w^{*}$ is the characteristic frequency of the medium.

\section{Solution of the Problem}

We assume the solutions of the form

$$
\left(u_{1}, u_{2}, T, \psi\right)=(1, W, R, S) U \exp \left\{i \xi\left(x_{1}+m x_{2}-c t\right)\right\},
$$

where $c=\omega / \xi$ is the nondimensional phase velocity, $\omega$ is the frequency, and $\xi$ is the wave number. $m$ is still unknown parameter. $1, W, R, S$ are, respectively, the amplitude ratios of $u_{1}, u_{2}, T$, and $u_{2}^{\ell}$ with respect to $u_{1}$. Substituting the values of $u_{1}, u_{2}$ and $T$ from (13) in (8), (9), and (10), we obtain

$$
\begin{aligned}
& {\left[\frac{m}{C_{11}}\left(C_{12}+C_{o}-\frac{P}{2}\right)\right] W+\left(\frac{-1}{i \xi}\right) R} \\
& +\left[1-c^{2}+\frac{1}{C_{11}}\left(C_{o}+\frac{P}{2}\right) m^{2}\right]=0, \\
& {\left[\frac{C_{22}}{C_{11}} m^{2}+\frac{1}{C_{11}}\left(C_{o}+\frac{P}{2}\right)-c^{2}\right] W} \\
& +\left(\frac{-m \bar{\beta}}{i \xi}\right) R+\left[\frac{1}{C_{11}}\left(C_{12}+C_{o}-\frac{P}{2}\right) m\right]=0, \\
& {\left[\varepsilon_{1} \bar{\beta}\left(-m c+m c^{2} \tau_{o} i \xi\right)\right] W+\left[1+\bar{k} m^{2}+\left(\frac{c}{i \xi}-\tau_{o} c^{2}\right)\right] R} \\
& \quad+\varepsilon_{1}\left[c-\tau_{o} c^{2} i \xi\right]=0,
\end{aligned}
$$

where

$$
\varepsilon_{1}=\frac{T_{o} \beta_{11}^{2}}{\rho w^{*} k_{11}} .
$$

The system of (14) has a nontrivial solution if the determinant of the coefficients $[1, W, R]^{T}$ vanishes, which yields to the following polynomial characteristic equation:

$$
m^{6}+d_{21} m^{4}+d_{31} m^{2}+d_{41}=0
$$

where

$$
\begin{gathered}
d_{1}=\left[\bar{k}\left(L_{2} g_{1}\right)\right], \\
d_{2}=\left[-g_{1} F L_{2}+\bar{k}\left(L_{1} g_{1}+L_{2} g_{2}-A_{1}^{2}\right)\right. \\
\left.+L_{2} g_{1}+(\bar{\beta})^{2} \varepsilon_{1} F L_{2}\right], \\
d_{3}=\left[-F g_{1}\left(L_{1}+\varepsilon_{1}\right)-F g_{2} L_{2}\right. \\
+\bar{\beta} F A_{1}\left(1-\varepsilon_{1}\right)+\bar{k}\left(L_{1} g_{2}\right)+L_{1} g_{1} \\
\left.+L_{2} g_{2}+(\bar{\beta})^{2} \varepsilon_{1} F L_{1}+A_{1}^{2}(F-1)\right], \\
d_{4}=\left[-g_{2} F\left(L_{1}+\varepsilon_{1}\right)+L_{1} g_{2}\right], \\
d_{21}=\frac{d_{2}}{d_{1}}, \quad d_{31}=\frac{d_{3}}{d_{1}}, \\
F=\frac{d_{41}=\frac{d_{4}}{d_{1}},}{i \xi}, \quad A=m A_{1}, \\
A_{1}=\frac{1}{C_{11}}\left(C_{12}+C_{o}-\frac{P}{2}\right), \\
G=g_{1} m^{2}+g_{2}, \quad g_{1}=\frac{C_{22}}{C_{11}}, \\
g_{2}=\frac{1}{C_{11}}\left(C_{o}+\frac{P}{2}\right)-c^{2}, \\
L=L_{1}+L_{2} m^{2}, \quad L_{1}=1-c^{2}, \\
L_{2}=\frac{1}{C_{11}}\left(C_{o}+\frac{P}{2}\right) .
\end{gathered}
$$

The characteristic equation (16) is cubic in $m^{2}$ and hence possesses three roots $\left(m_{p}^{2}, p=1,2,3\right)$. Therefore, there exist three types of quasi-waves in transversely isotropic elastic half-space, namely, quasi-longitudinal waves $(\mathrm{QP})$, quasitransverse waves (QSV), and quasi-thermal waves (QT).

The formal expression for displacements and volume fraction field, satisfying the radiation condition that $\operatorname{Re}\left(m_{p}\right) \geq$ 0 , can be written as

$$
\left(u_{1}, u_{2}, T\right)=\sum_{p=1}^{3} G_{p}\left(1, n_{1 p}, n_{2 p}\right) \exp \left\{i \xi\left(x_{1}+i m_{p} x_{2}-c t\right)\right\},
$$

where

$$
\begin{gathered}
n_{1 p}=\frac{\Delta_{1}}{\Delta}, \quad n_{2 p}=\frac{\Delta_{2}}{\Delta}, \\
\Delta_{1}=\left(\frac{-\bar{\beta} m \varepsilon_{1} c\left(1-\tau_{o} c i \xi\right)}{i \xi}-A Y\right), \\
\Delta_{2}=\left(G \varepsilon_{1} c\left(1-\tau_{o} c i \xi\right)-A Z\right),
\end{gathered}
$$




$$
\begin{gathered}
\Delta=\left(G Y+\frac{\bar{\beta} m Z}{i \xi}\right), \\
Y=1+\bar{k} m^{2}+\left(\frac{c}{i \xi}-\tau_{o} c^{2}\right), \\
Z=\varepsilon_{1} \bar{\beta} m c\left(-1+c \tau_{o} i \xi\right)
\end{gathered}
$$

and $G_{1}, G_{2}, G_{3}$ are arbitrary constants.

Substituting the values of $\psi$ from (13) in (5) and after simplification, we obtain

$$
\psi=\left[G_{4} \sin \left(\xi m_{4} x_{2}\right)+G_{5} \cos \left(\xi m_{4} x_{2}\right)\right] \exp \left(i \xi\left(x_{1}-c t\right)\right),
$$

where

$$
m_{4}= \pm\left(\left(\frac{v_{1} c}{\alpha^{\ell}}\right)^{2}-1\right)^{1 / 2}
$$

and $G_{4}, G_{5}$ are arbitrary constants.

The normal displacement and normal stress for medium $M_{2}$, with the help of (5), (6), (12), and (20), are given by

$$
\begin{aligned}
u_{2}^{\ell}= & m_{4} \xi\left[G_{4} \cos \left(\xi m_{4} x_{2}\right)-G_{5} \sin \left(\xi m_{4} x_{2}\right)\right] \\
& \times \exp \left(i \xi\left(x_{1}-c t\right)\right), \\
t_{22}^{\ell}= & -\xi^{2}\left(1+m_{4}^{2}\right)\left[G_{4} \sin \left(\xi m_{4} x_{2}\right)+G_{5} \cos \left(\xi m_{4} x_{2}\right)\right] \\
& \times \exp \left(i \xi\left(x_{1}-c t\right)\right) .
\end{aligned}
$$

Also from (3) we have

$$
\begin{gathered}
t_{22}=C_{12} u_{1,1}+C_{22} u_{2,2}-\beta_{22} T, \\
t_{21}=C_{o}\left(u_{1,2}+u_{2,1}\right) .
\end{gathered}
$$

\section{Boundary Conditions}

The appropriate boundary conditions are as follows:

(i) vanishing of normal stress component at the free surface of the liquid layer, $x_{2}=-H$; that is,

$$
\left(t_{22}^{\ell}\right)_{M_{2}}=-p^{\ell}=0
$$

(ii) continuity of normal stress components at the interface $x_{2}=0$; that is,

$$
\left(t_{22}\right)_{M_{1}}=-p^{\ell},
$$

(iii) normal displacement components at the interface $x_{2}=0$; that is,

$$
\left(u_{2}^{\ell}\right)_{M_{2}}=\left(\dot{u}_{2}\right)_{M_{1}}
$$

(iv) vanishing shear stress for medium $M_{1}$ at the interface $x_{2}=0$; that is,

$$
\left(t_{21}\right)_{M_{1}}=0,
$$

(v) thermal boundary conditions

$$
\frac{\partial T}{\partial x_{2}}+h T=0,
$$

where $h \longrightarrow 0$ corresponds Insulated boundary condition, $h \longrightarrow \infty$ corresponds Isolated boundary condition.

\section{Derivations of the Secular Equations}

Substituting the value of $u_{2}, T, u_{2}^{\ell}, t_{22}^{\ell}$ from (18) and (22) in (24) $-(28)$ and with the aid of (7) and (12), after simplification, we obtain

$$
c^{2}=\left(\frac{\alpha^{\ell}}{v_{1}}\right)^{2}\left[\frac{\left(\tan ^{-1}\left(T_{11}\right)\right)^{2}+\xi^{2} H^{2}}{\xi^{2} H^{2}}\right],
$$

where

$$
\begin{gathered}
T_{11}=\frac{C_{11} m_{4}\left(E_{11} D_{1}-E_{12} D_{2}+E_{13} D_{3}\right)}{i c \rho^{\ell} v_{1}^{2}\left(n_{11} D_{1}-n_{12} D_{2}+n_{13} D_{3}\right)}, \\
E_{1 p}=i \xi\left(\frac{C_{12}}{C_{11}}\right)+\left(\frac{C_{22}}{C_{11}}\right) n_{1 p}\left(i^{2} \xi m_{p}\right)-\left(\frac{\beta_{22}}{\beta_{11}}\right) n_{2 p}, \\
E_{2 p}=\xi\left(n_{1 p} i-m_{p}\right), \quad E_{3 p}=n_{2 p}\left(h-\xi m_{p}\right), \\
\quad(p=1,2,3), \\
D_{1}=E_{22} E_{33}-E_{32} E_{23}, \quad D_{2}=E_{21} E_{33}-E_{31} E_{23}, \\
D_{3}=E_{21} E_{32}-E_{31} E_{22} .
\end{gathered}
$$

Equation (29) is surface waves secular equation of fiberreinforced transversely isotropic thermoelastic half-space under a uniform homogeneous inviscid liquid layer. The secular equation has complete information about the phase velocity, wave number, and attenuation coefficient of surface waves in such medium.

\subsection{Particular Cases}

(i) If we take $h \rightarrow 0$ in (29), we obtain the frequency equation for the thermally insulated boundary.

(ii) If we take $h \rightarrow \infty$ in (29), we obtain the frequency equation for the thermally isolated boundary.

(iii) Taking $P \rightarrow 0$ in secular equation (29), yield the frequency equation for fiber-reinforced transversely isotropic thermoelastic half space without initial stress under a uniform homogeneous inviscid liquid layer.

(iv) For $\mu_{T}=\mu_{L}=\mu, K_{11}=K_{22}=K, \beta_{11}=\beta_{22}=\beta=$ $(3 \lambda+2 \mu) \alpha_{t}$, (29) reduces to the frequency equation in a homogeneous Isotropic half space under inviscid liquid.

(v) $\tau_{o} \rightarrow 0$ in (10), we obtained the frequency equation in fiber-reinforced coupled thermoelastic half space under inviscid liquid layer. 


\section{Rayleigh Wave}

(1) Taking $H \rightarrow 0$, the frequency equation (29) reduces to

$$
\begin{gathered}
E_{11}\left(E_{22} E_{33}-E_{23} E_{32}\right)-E_{12}\left(E_{21} E_{33}-E_{31} E_{23}\right) \\
+E_{13}\left(E_{21} E_{32}-E_{31} E_{22}\right)=0 .
\end{gathered}
$$

Equation (31) is the frequency equation for Rayleigh surface wave propagation in a fiber-reinforced transversely isotropic thermoelastic half-space with initial stress. In general wave number and hence phase velocity of waves is complex quantity therefore the waves are attenuated in space. If we write

$$
c^{-1}=v^{-1}+i \omega^{-1} F
$$

so that, $\xi=K+i F$, where $K=\omega / v, v$ and $F$ are real. Also the roots of characteristic equation (16) are in general complex and hence we assume that $m_{p}=p_{p}+i q_{p}$, so that the exponent in the plane wave solutions (18) for the half-space becomes

$$
i K\left(x_{1}-m_{p}^{I} x_{2}-v t\right)-K\left(\frac{F}{K} x_{1}+m_{p}^{R} x_{2}\right)
$$

where

$$
\begin{array}{r}
m_{p}^{R}=p_{p}-q_{p} \frac{F}{K}, \quad m_{p}^{I}=q_{p}+p_{p} \frac{F}{K}, \\
(p=1,2,3) .
\end{array}
$$

This shows that $v$ is the propagation velocity and $F$ is the attenuation coefficient of wave.

The equation (18) can be rewritten as

$$
\begin{aligned}
& u_{1}=\sum_{p=1}^{3} G_{p} \exp \left(-F x_{1}-\lambda_{p}^{R} x_{2}\right) \\
& \quad \times \exp \left[i\left(K\left(x_{1}-v t\right)-\lambda_{p}^{I} x_{2}\right)\right], \\
& u_{2}=\sum_{p=1}^{3} n_{1 p} G_{p} \exp \left(-F x_{1}-\lambda_{p}^{R} x_{2}\right) \\
& \quad \times \exp \left[i\left(K\left(x_{1}-v t\right)-\lambda_{p}^{I} x_{2}\right)\right], \\
& T=\sum_{p=1}^{3} n_{2 p} G_{p} \exp \left(-F x_{1}-\lambda_{p}^{R} x_{2}\right) \\
& \quad \times \exp \left[i\left(K\left(x_{1}-v t\right)-\lambda_{p}^{I} x_{2}\right)\right]
\end{aligned}
$$

with $\lambda_{p}=K\left(m_{p}^{R}+i m_{p}^{I}\right)=\lambda_{p}^{R}+i \lambda_{p}^{I}$ (say), $(p=1,2,3)$. Moreover it is clear that

$$
\left|\lambda_{p}^{R}\right|^{2}-\left|\lambda_{p}^{I}\right|^{2}=K^{2}\left[\left(m_{p}^{R}\right)^{2}-\left(m_{p}^{I}\right)^{2}\right]
$$

7.1. Normal Stress. Normal stress at the interface $x_{2}=0$ is given by

$$
\begin{aligned}
t_{22}= & \left(C_{12} i \xi \sum_{p=1}^{3} G_{p}-C_{22} \xi \sum_{p=1}^{3} m_{p} G_{p} n_{1 p}-\beta_{22} \sum_{p=1}^{3} G_{p} n_{2 p}\right) \\
& \times \exp \left(i \xi\left(x_{1}-v t\right)\right), \quad(p=1,2,3) .
\end{aligned}
$$

7.2. Specific Loss. The Specific loss is the ratio of energy $(\Delta W)$ dissipated in taking a specimen through a stress cycle, to the elastic energy $(W)$ stored in the specimen when the strain is maximum. The Specific loss is the most direct method of defining internal friction for a material. For a sinusoidal plane wave of small amplitude, Kolsky [32] shows that the specific loss $\Delta W / W$ equals $4 \pi$ times the absolute value of imaginary part of $\xi$ to the real part of $\xi$; that is,

$$
\frac{\Delta W}{W}=4 \pi\left|\frac{\operatorname{Im}(\xi)}{\operatorname{Re}(\xi)}\right|=4 \pi\left|\frac{v G}{\omega}\right|=4 \pi\left|\frac{G}{F}\right| .
$$

\section{Numerical Results and Discussion}

For numerical computations, we take the following values of the relevant parameters for generalized fiber-reinforced transversely isotropic thermoelastic solid:

$$
\begin{gathered}
\lambda=5.66 \times 10^{10} \mathrm{~N} / \mathrm{m}^{2}, \quad \rho=2660 \mathrm{Kg} / \mathrm{m}^{3}, \\
\mu_{T}=2.46 \times 10^{10} \mathrm{~N} / \mathrm{m}^{2}, \quad \mu_{L}=5.66 \times 10^{10} \mathrm{~N} / \mathrm{m}^{2}, \\
\alpha=-1.28 \times 10^{10} \mathrm{~N} / \mathrm{m}^{2}, \quad \beta=220.90 \times 10^{10} \mathrm{~N} / \mathrm{m}^{2}, \\
K_{11}=0.0921 \times 10^{3} \mathrm{Jm}^{-1} \mathrm{deg}^{-1} \mathrm{~s}^{-1}, \quad P=100 \\
K_{22}=0.0963 \times 10^{3} \mathrm{Jm}^{-1} \mathrm{deg}^{-1} \mathrm{~s}^{-1}, \\
\alpha_{1}=0.017 \times 10^{4} \mathrm{deg}^{-1}, \\
\alpha_{2}=0.015 \times 10^{4} \mathrm{deg}^{-1}, \quad T_{o}=293 \mathrm{~K}, \\
\tau_{o}=0.05 \mathrm{~s}^{-1} \quad h=1, \quad H=1, \\
C_{e}=0.787 \times 10^{3} \mathrm{JKg}^{-1} \mathrm{deg}^{-1}, \quad \omega=2 \mathrm{~s}^{-1} .
\end{gathered}
$$
[31]:

The physical constants for water are given by Ewing et al.

$$
\rho^{\ell}=1.0 \times 10^{3} \mathrm{Kg} / \mathrm{m}^{3}, \quad \lambda^{\ell}=2.14 \times 10^{3} \mathrm{~N} / \mathrm{m}^{2} .
$$

For comparison with the generalized fiber-reinforced isotropic thermoelastic solid following Ezzat [33], we take 


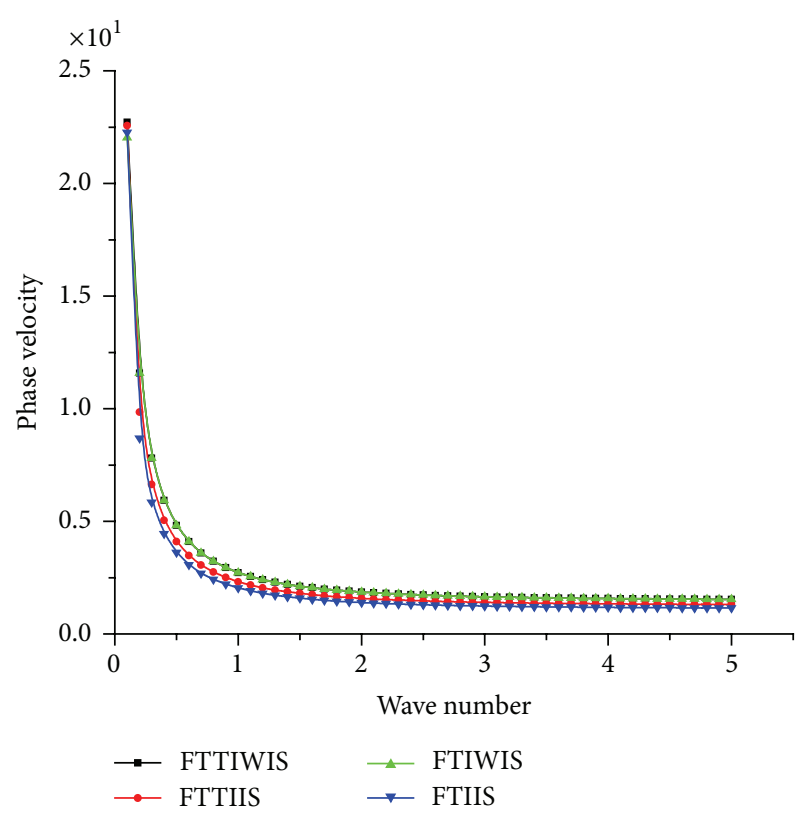

FIGURE 2: Variation of phase velocity w.r.t. wave number (with and without initial stress).

the following values of relevant parameters for the case of copper material as

$$
\begin{gathered}
\lambda=7.76 \times 10^{10} \mathrm{~N} / \mathrm{m}^{2}, \\
\mu_{T}=\mu_{L}=\mu=3.86 \times 10^{10} \mathrm{~N} / \mathrm{m}^{2}, \\
K_{11}=K_{22}=K=386 \mathrm{NK}^{-1} \mathrm{~s}^{-1}, \\
\beta_{11}=\beta_{22}=\beta=(3 \lambda+2 \mu) \alpha_{t}, \\
\alpha_{t}=1.78 \times 10^{-5} \mathrm{~K}^{-1} \\
\alpha_{1}=1.005 \mathrm{deg}^{-1}, \quad \alpha_{2}=1.001 \mathrm{deg}^{-1}, \\
C_{e}=383.1 \mathrm{~m}^{2} \mathrm{~K}^{-1} \mathrm{~s}^{-1}, \quad P=0, \\
H=1, \quad \rho=8954 \mathrm{Kg} / \mathrm{m}^{3}, \\
\tau_{o}=0.1 \mathrm{~s}, \quad h=1 .
\end{gathered}
$$

Using the above values of parameters, the dispersion curves of nondimensional phase velocity, attenuation coefficient and specific heat are shown graphically with respect to nondimensional wave number. Here, we consider abbreviations FTTIWIS, FTTIIS, FTIWIS, and FTIIS for fiberreinforced transversely isotropic thermoelastic material without initial stress, fiber-reinforced transversely isotropic thermoelastic material with initial stress, fiber-reinforced isotropic thermoelastic material without initial stress, and fiberreinforced isotropic thermoelastic material with initial stress respectively and $H$ refers to the thickness of the layer.

Figure 2 depicts the variations of phase velocity with respect to the wave number. The behavior of phase velocity

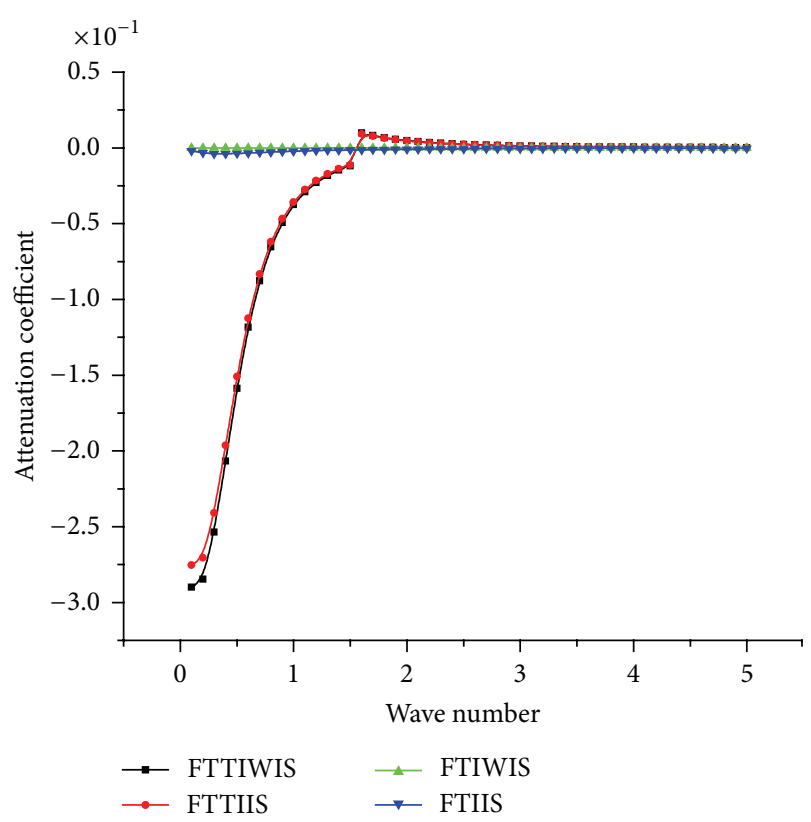

FIGURE 3: Variation of attenuation coefficient w.r.t. wave number (with and without initial stress).

for FTTIWIS, FTTIIS, FTIWIS, and FTIIS is similar but the corresponding values are different in magnitude. The values of phase velocity decrease for lower wave number and become stable for higher wave number in all the cases. Figure 3 shows the variation of attenuation coefficient with respect to the wave number. For FTTIWIS and FTTIIS the values of attenuation coefficient increase monotonically up to wave number $(\xi)=2$ and after that it becomes constant for whole range of wave number. On the other hand, the values of attenuation coefficients are consistent within the whole range of wave number for FTIWIS and FTIIS.

Figure 4 indicates the variation of phase velocity with respect to the wave number for different values of thickness of layer. Here, we consider the particular case of fiber-reinforced transversely isotropic thermoelastic material and analyses of the behavior of curves with respect to initial stress. It is observed that for small value of wave number the graph of phase velocity shows opposite behavior w.r.t. thickness, that is, on increasing the values of thickness of layer, the values of corresponding phase velocity decreases, and it became steady for large values of wave number.

Figure 5 shows the variation of attenuation coefficient with respect to the wave number for different value of thickness of the layer. Here also we considered the particular case of fiber-reinforced transversely isotropic thermoelastic material and analyses of the behavior of curves with respect to initial stress. It is perceived that on decreasing the value of $H$ the curve shows consistency for small value of wave number and on increasing the value of $H$ the curve shows uniformity for large value of $\xi$.

Figure 6 depicts the variations of specific loss verses wave number. For FTIWIS and FTIIS the curve shows sharp decrease in the value of specific loss for the range $0 \leq \xi \leq 1$ and become parallel for $\xi>1$. It is observed that graphs 


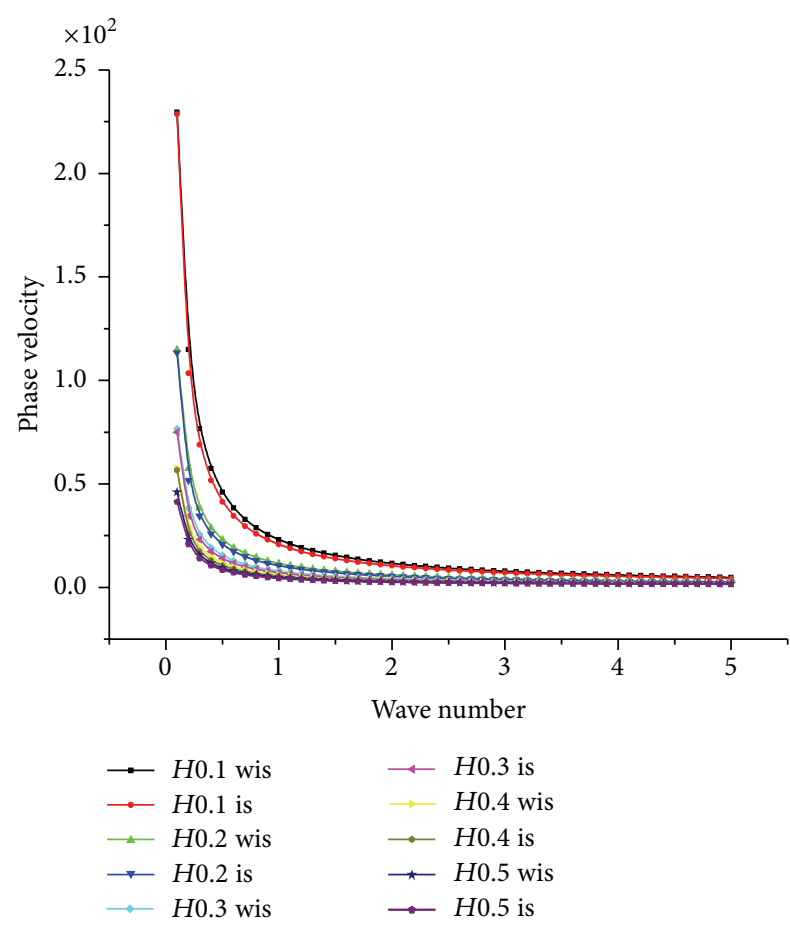

FIGURE 4: Variation of phase velocity w.r.t. wave number (with and without initial stress for different value of $H$ ).

corresponding to FTTIWIS and FTTIIS follow the similar pattern for whole range of $\xi$ but the values are different in magnitude.

8.1. Rayleigh Wave. Figure 7 shows the variations of phase velocity of Rayleigh wave verses wave number for FTTIWIS, FTTIIS, FTIWIS, and FTIIS. The value of phase velocity decreases for small values of $\xi$ and become stable for higher value of $\xi$. It is analyzed that in all the cases behavior of curves are almost the same within the range $2 \leq \xi \leq 5$. Figure 8 shows the variations of attenuation coefficient of Rayleigh wave verses wave number for FTTIWIS, FTTIIS, FTIWIS, and FTIIS. For FTIWIS and FTIIS the graph shows a sudden fall for $\xi=0.5$ and then increases monotonically up to $\xi=2$ and becomes constant for the remaining range of $\xi$. A large difference between the behavior of curves for the transversely isotropic and isotropic case is also perceived.

\section{Conclusions}

The propagation of surface waves in an initially stressed fiber-reinforced transversely isotropic thermoelastic material under an inviscid liquid layer has been studied. After developing the formal solutions, the secular equation for surface wave propagation is derived. Some special cases of frequency equation are also discussed. The attenuation coefficients and specific loss (liquid layer and half-space) have also been studied and computed for FTTIWIS, FTTIIS, FTIWIS, and FTIIS. The trend of phase velocity is the same in all these four cases for surface wave but varies for attenuation

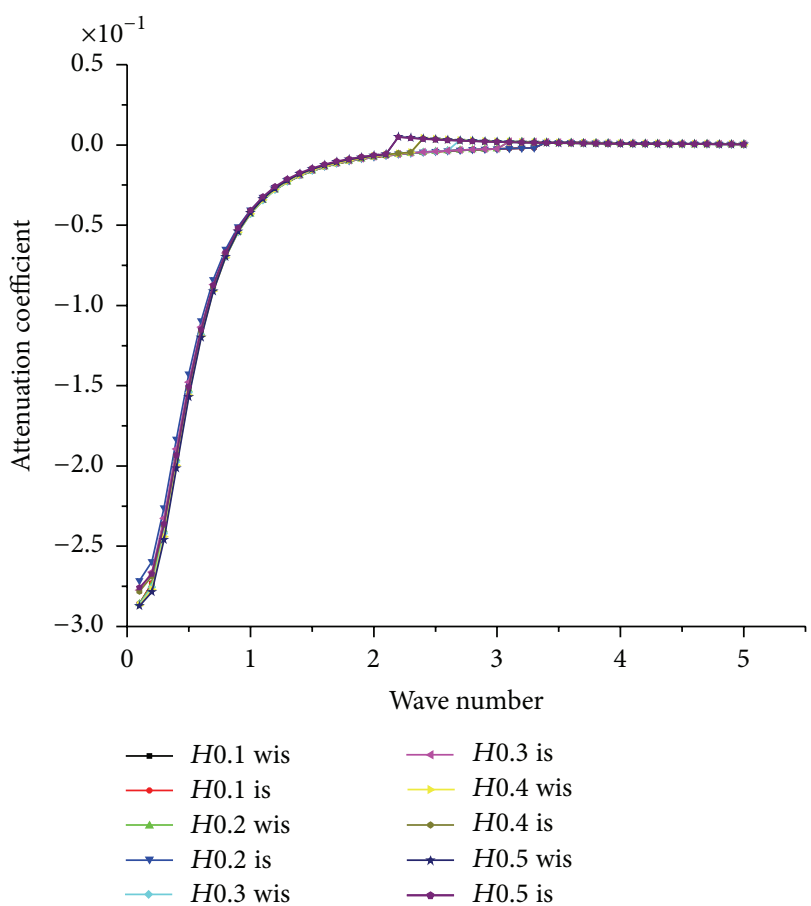

FIGURE 5: Variation of attenuation coeff. w.r.t. wave number (with and without initial stress for different value of $H$ ).

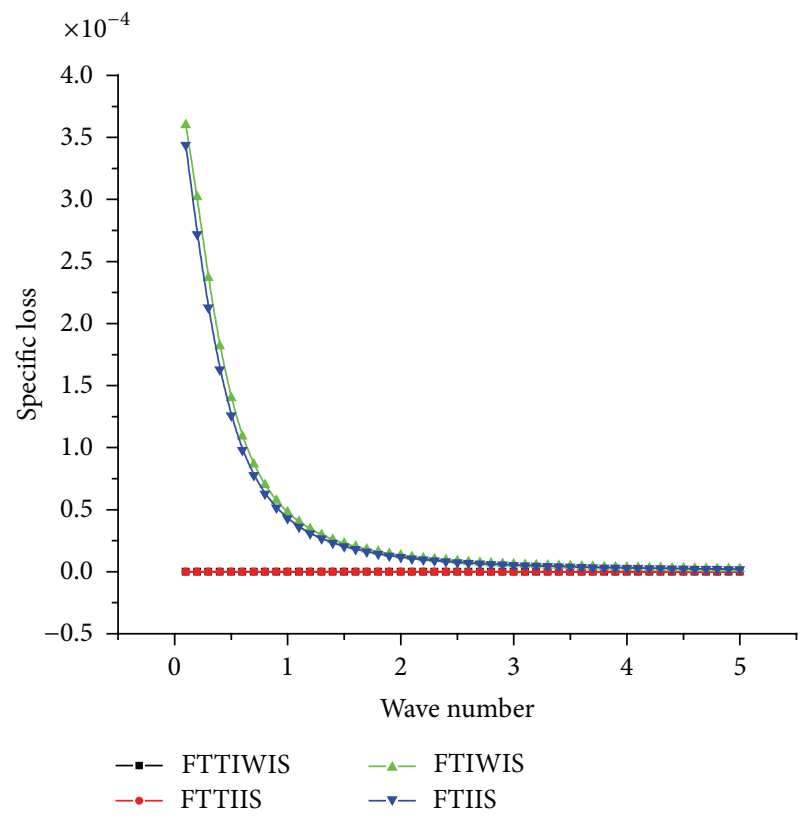

Figure 6: Variation of specific loss w.r.t. wave number (with and without initial stress).

coefficient. From numerical analysis, it is shown that the phase speeds and attenuation coefficients for Rayleigh wave are also affected significantly due to the presence of initial stress parameters. 


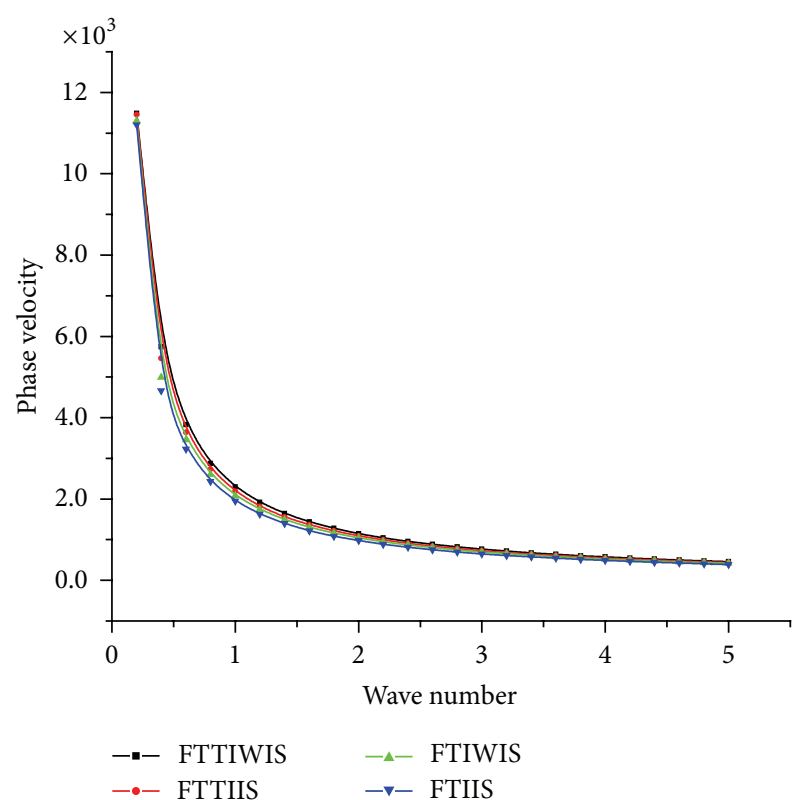

FIGURE 7: Variation of phase velocity w.r.t. wave number for Rayleigh wave (with and without initial stress).

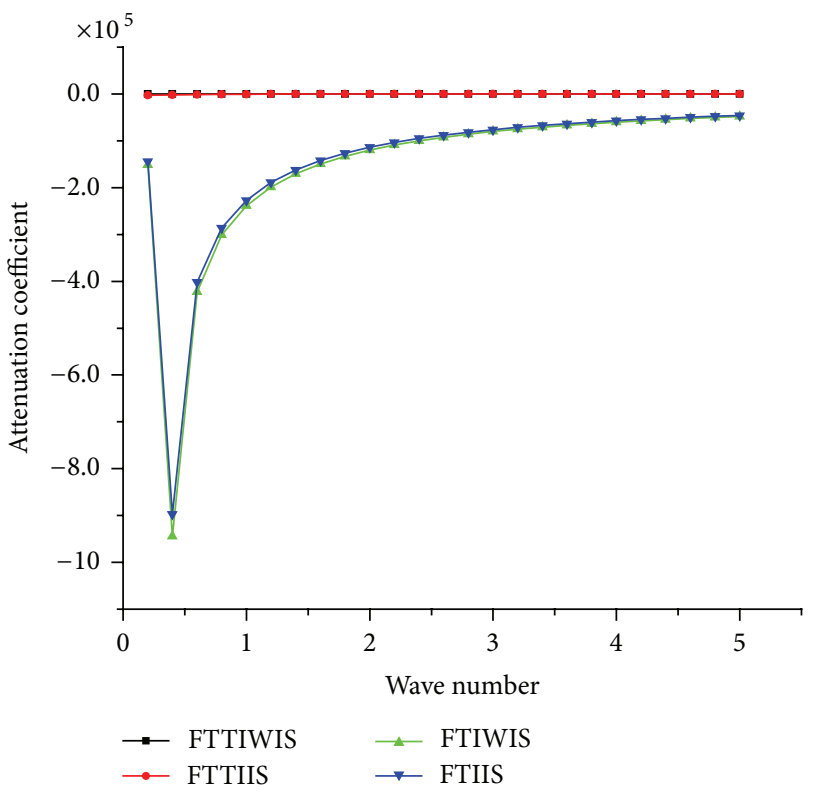

FIGURE 8: Variation of attenuation coeff. w.r.t. wave number for Rayleigh wave (with and without initial stress).

\section{Conflict of Interests}

The authors declare that there is no conflict of interests regarding the publication of this paper.

\section{References}

[1] A. J. M. Spencer, Deformation of Fibre-reinforced Materials, University of Oxford, Clarendon, Va, USA, 1941.
[2] A. J. Belfield, T. G. Rogers, and A. J. M. Spencer, "Stress in elastic plates reinforced by fibres lying in concentric circles," Journal of the Mechanics and Physics of Solids, vol. 31, no. 1, pp. 25-54, 1983.

[3] A. C. Pipkin, "Finite deformations of ideal fiber-reinforced composites," in Composites Materials, G. P. Sendeckyj, Ed., vol. 2 of Mechanics of Composite Materials, pp. 251-308, Academic Press, New York, NY, USA, 1973.

[4] P. R. Sengupta and S. Nath, "Surface waves in fibre-reinforced anisotropic elastic media," Indian Academy of Sciences, Sadhana Proceedings, vol. 26, pp. 363-370, 2001.

[5] H. W. Lord and Y. Shulman, "A generalized dynamical theory of thermoelasticity," Journal of the Mechanics and Physics of Solids, vol. 15, no. 5, pp. 299-309, 1967.

[6] R. S. Dhaliwal and H. H. Sherief, "Generalized thermoelasticity for anisotropic media," Quarterly of Applied Mathematics, vol. 38, no. 1, pp. 1-8, 1980/81.

[7] A. U. Erdem, "Heat Conduction in fiber-reinforced rigid bodies," 10 Ulusal Ist Bilimi ve Tekmgi Kongrest (ULIBTK), 6-8 Eylul, Ankara, Turkey, 1995.

[8] R. Kumar and R. R. Gupta, "Study of wave motion in an anisotropic fiber-reinforced thermoelastic solid," Journal of Solid Mechanics, vol. 2, no. 1, pp. 91-100, 2010.

[9] P. Chadwick and L. T. C. Seet, "Wave propagation in a transversely isotropic heat-conducting elastic material," Mathematika, vol. 17, pp. 255-274, 1970.

[10] H. Singh and J. N. Sharma, "Generalized thermoelastic waves in transversely isotropic media," Journal of the Acoustical Society of America, vol. 77, pp. 1046-1053, 1985.

[11] B. Singh, "Wave propagation in an anisotropic generalized thermoelastic solid," Indian Journal of Pure and Applied Mathematics, vol. 34, no. 10, pp. 1479-1485, 2003.

[12] P. Chadwick, Progress in Solid Mechanics, vol. 1, North-Holland, Amsterdam, The Netherlands, 1960, edited by R. Hill, I. N. Sneddon.

[13] P. Chadwick and D. W. Windle, "Propagation of Rayleigh waves along isothermal and insulated boundaries," Proceedings of the Royal Society of America, vol. 280, pp. 47-71, 1964.

[14] J. N. Sharma and H. Singh, "Thermoelastic surface waves in a transversely isotropic half space with thermal relaxations," Indian Journal of Pure and Applied Mathematics, vol. 16, no. 10, pp. 1202-1219, 1985.

[15] A. Montanaro, "On singular surfaces in isotropic linear thermoelasticity with initial stress," Journal of the Acoustical Society of America, vol. 106, no. 3, pp. 1586-1588, 1999.

[16] J. Wang and P. Slattery, "Thermoelasticity without energy dissipation for initially stressed bodies," International Journal of Mathematics and Mathematical Sciences, vol. 31, no. 6, pp. 329337, 2002.

[17] D. Ieşan, "A theory of prestressed thermoelastic Cosserat continua," Journal of Applied Mathematics and Mechanics, vol. 88, no. 4, pp. 306-319, 2008.

[18] K. Ames and B. Straughan, "Continuous dependence results for initially prestressed thermoelastic bodies," International Journal of Engineering Science, vol. 30, no. 1, pp. 7-13, 1992.

[19] J. Wang, R. S. Dhaliwal, and S. R. Majumdar, "Some theorems in the generalized theory of thermoelasticity for prestressed bodies," Indian Journal of Pure and Applied Mathematics, vol. 28, no. 2, pp. 267-276, 1997.

[20] M. Marin and C. Marinescu, "Thermoelasticity of initially stressed bodies, asymptotic equipartition of energies," International Journal of Engineering Science, vol. 36, no. 1, pp. 73-86, 1998. 
[21] V. V. Kalinchuk, T. I. Belyankova, Y. E. Puzanoff, and I. A. Zaitseva, "Some dynamic properties of the nonhomogeneous thermoelastic prestressed media," The Journal of the Acoustical Society of America, vol. 105, no. 2, p. 1342, 1999.

[22] M. I. A. Othman and Y. Song, "Reflection of plane waves from an elastic solid half-space under hydrostatic initial stress without energy dissipation," International Journal of Solids and Structures, vol. 44, no. 17, pp. 5651-5664, 2007.

[23] B. Singh, "Wave propagation in an initially stressed transversely isotropic thermoelastic solid half-space," Applied Mathematics and Computation, vol. 217, no. 2, pp. 705-715, 2010.

[24] M. I. A. Othman, S. M. Said, and N. Sarker, "Effect of hydrostatic initial stress on a fiber-reinforced thermoelastic medium with fractional derivative heat transfer," Multidiscipline Modeling in Materials and Structures, vol. 9, no. 3, pp. 410-426, 2013.

[25] A. M. Abd-Alla, S. M. Abo-Dahab, and A. Al-Mullise, "effects of rotation and gravity field on surface waves in fibre-reinforced thermoela stic media under four theories," Journal of Applied Mathematics, vol. 2013, Article ID 562369, 10 pages, 2013.

[26] M. I. A. Othman and S. Y. Atwa, "Effect of rotation on a fiber-reinforced thermo-elastic under Green-Naghdi theory and influence of gravity," Meccanica, vol. 49, no. 1, pp. 23-36, 2014.

[27] I. A. Abbas, "Generalized magneto-thermoelastic interaction in a fiber-reinforced anisotropic hollow cylinder," International Journal of Thermophysics, vol. 33, no. 3, pp. 567-579, 2012.

[28] I. A. Abbas, "A GN model for thermoelastic interaction in an unbounded fiber-reinforced anisotropic medium with a circular hole," Applied Mathematics Letters, vol. 26, no. 2, pp. 232-239, 2013.

[29] I. A. Abbas and A. Zenkour, "Two-temperature generalized thermoelastic interaction in an infinite fiber-reinforced anisotropic plate containing a circular cavity with two relaxation times," Journal of Computational and Theoretical Nanoscience, vol. 11, no. 1, pp. 1-7, 2014.

[30] I. A. Abbas and M. I. A. Othman, "Generalized thermoelastic interaction in a fiber-reinforced anisotropic half-space under hydrostatic initial stress," Journal of Vibration and Control, vol. 18, no. 2, pp. 175-182, 2012.

[31] W. M. Ewing, W. S. Jardetzky, and F. Press, Elastic Waves in Layered Media, McGraw-Hill, New York, NY, USA, 1957.

[32] H. Kolsky, Stress Waves in Solids, Clarendon Press, Dover, New York, NY, USA, 1963.

[33] M. A. Ezzat, "Fundamental solution in generalized magnetothermoelasticity with two relaxation times for perfect conductor cylindrical region," International Journal of Engineering Science, vol. 42, no. 13-14, pp. 1503-1519, 2004. 

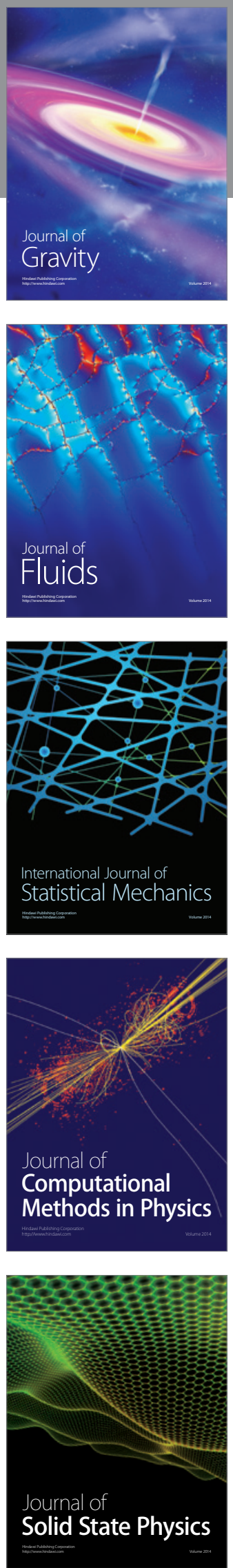

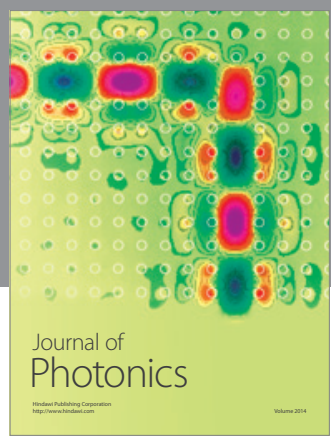

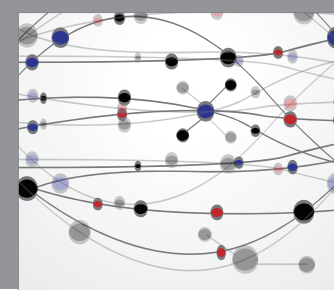

The Scientific World Journal

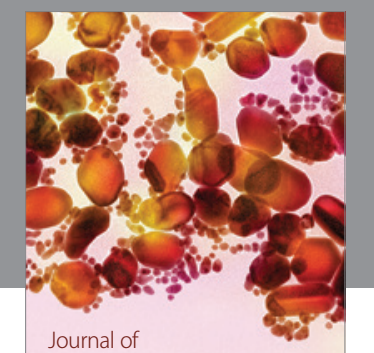

Soft Matter
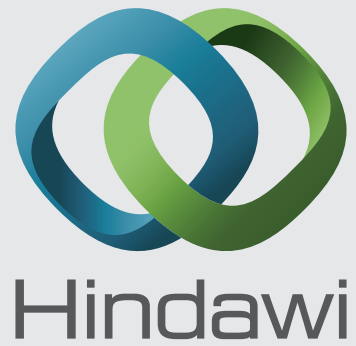

Submit your manuscripts at

http://www.hindawi.com
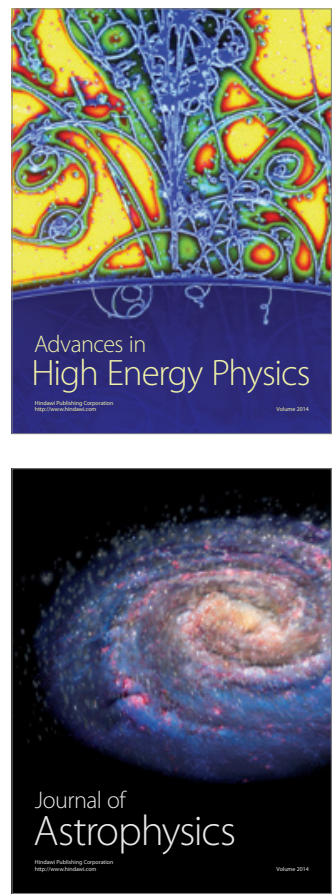
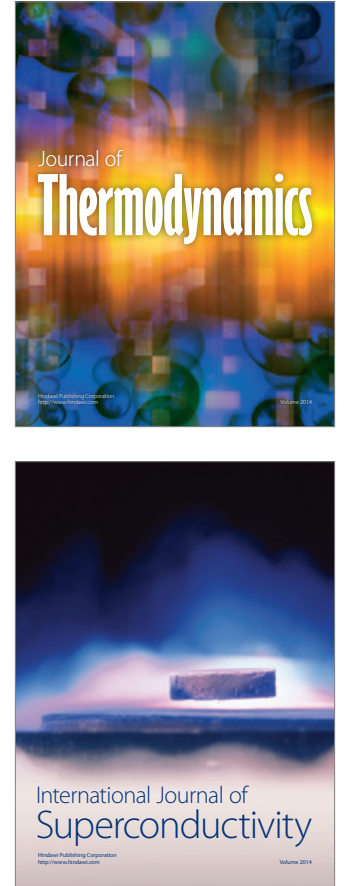
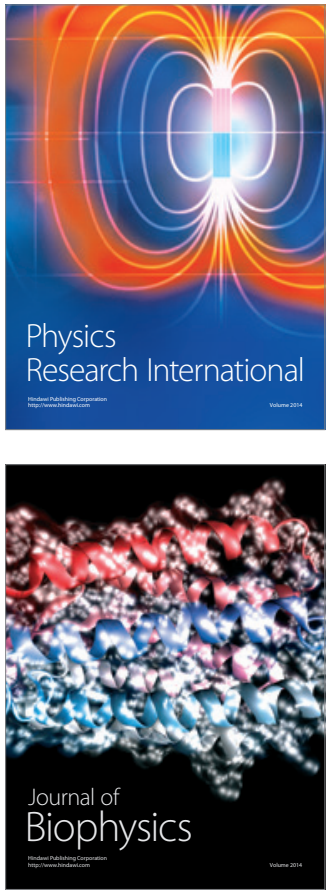
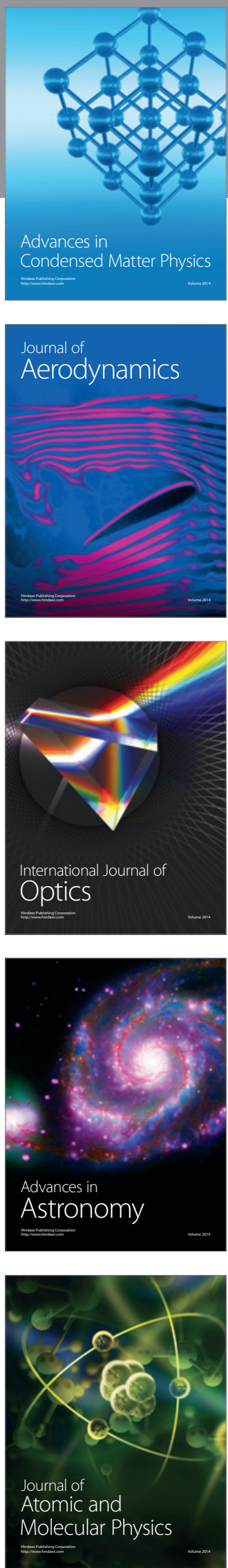